\title{
Ultralow Threshold One-Photon- and Two-Photon-Pumped Optical Gain Media of Blue-Emitting Colloidal Quantum Dot Films
}

\author{
Burak Guzelturk, ${ }^{\dagger, \ddagger}, \#$ Yusuf Kelestemur, ${ }^{\dagger, \#}$ Mehmet Zafer Akgul, ${ }^{\dagger, \#}$ Vijay Kumar Sharma, ${ }^{\dagger}$ \\ and Hilmi Volkan Demir ${ }^{*}, \dagger$
}

\begin{abstract}
${ }^{\dagger}$ Department of Electrical and Electronics Engineering, Department of Physics and UNAM - Institute of Materials Science and Nanotechnology, Bilkent University, Ankara 06800, Turkey

${ }^{\ddagger}$ Luminous! Center of Excellence for Semiconductor Lighting and Displays, School of Electrical and Electronic Engineering, School of Physical and Materials Sciences, Nanyang Technological University, Singapore 639798, Singapore
\end{abstract}

\section{Supporting Information}

ABSTRACT: Colloidal quantum dots (QDs) offer advantageous properties as an optical gain media for lasers. Optical gain in the QDs has been shown in the whole visible spectrum, yet it has been intrinsically challenging to realize efficient amplified spontaneous emission (ASE) and lasing in the blue region of the visible spectrum. Here, we synthesize large-sized core/gradient shell $\mathrm{CdZnS} / \mathrm{ZnS}$ QDs as an efficient optical gain media in the blue spectral range. In this Letter, we demonstrate for the first time that two-photon-absorption-pumped ASE from the blue-emitting QD is achievable with a threshold as low as $6 \mathrm{~mJ} / \mathrm{cm}^{2}$. Utilizing these QDs, we also report one-photonabsorption-pumped ASE at an ultralow threshold of $\sim 60 \mu \mathrm{J} / \mathrm{cm}^{2}$, which is comparable to the state-of-the-art red-emitting QD-based gain media. This one-photon-pumped ASE threshold is an order of magnitude better than that of the previously reported best blue-emitting QD-based gain media.

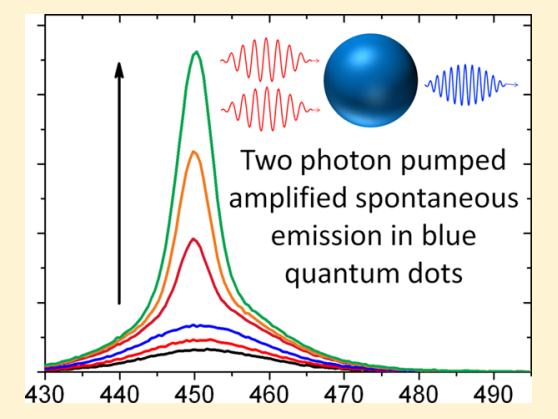

SECTION: Physical Processes in Nanomaterials and Nanostructures

$\mathrm{C}$ olloidal quantum dots (QDs) are favorable light-emitting materials owing to their size, shape, and composition tailorable optical properties, ${ }^{1,2}$ which make them promising light-emitting materials for light-emitting diodes ${ }^{3}$ and lasers. ${ }^{4-8}$ QD-based optical gain media represent an important field of study because their emission spectrum is easily tunable and full color lasing is possible with potentially high gain, which make them competitive against other gain media such as organic materials or other quantum-confined structures. ${ }^{8-10}$ Recently, full color lasing was successfully demonstrated in the QD-based gain media by one-photon absorption (1PA) pumping. ${ }^{8}$ Also, two-photon absorption pumping (2PA) was shown to be an alternative route for the excitation of the $\mathrm{QD}$-based optical gain media for frequency up-converted lasing. ${ }^{11-17}$ However, for the blue region of the visible spectrum, optical gain performance of the QD-based gain media has been severely limited as compared to the other color-emitting QDs (i.e., green, yellow and red). Up to this date, several reports demonstrated amplified spontaneous emission (ASE) in the blue region only with 1PA pumping. ${ }^{6,8,18,19}$ The first demonstration of the ASE in the blue region was achieved only at cryogenic temperatures. ${ }^{18}$ Later, Bawendi et al. demonstrated the first roomtemperature ASE and laser by blue-emitting QDs. ${ }^{6}$ To the best of our knowledge, the lowest ASE threshold for the blueemitting QDs was shown to be $800 \mu \mathrm{J} / \mathrm{cm}^{2} .{ }^{8}$ Additionally, 2PApumped ASE of blue-emitting QDs was not possible before. This poor optical gain performance of the blue-emitting QDs is probably due to their small size, where strong nonradiative
Auger recombination (AR) is expected to dominate and create ample losses. In addition, it was demonstrated that the optical gain threshold is size-independent provided that the hot exciton surface trapping is minimized..$^{20-23}$ In addition, the small size of these QDs had restrained the 2PA cross section, preventing achieving 2PA-pumped optical gain.

In this work, we developed $\mathrm{CdZnS} / \mathrm{ZnS}$ having both a large size and gradient shell with a mean size in the range of $8-10$ $\mathrm{nm}$. These QDs have emission in the blue region of 440-460 $\mathrm{nm}$. We demonstrate high-efficiency 1PA- and 2PA-pumped ASE employing these QDs as the optical gain media. The 1PApumped ASE threshold is as low as $60 \mu \mathrm{J} / \mathrm{cm}^{2}$, which represents more than an order of magnitude enhancement as compared to that of the blue-emitting QDs, as reported before. In addition, we report for the first time that 2PA-pumped ASE of blue-emitting QDs is viable, and we achieve an ASE threshold of $6 \mathrm{~mJ} / \mathrm{cm}^{2}$, which is comparable to that of the 2PApumped large-size red-emitting QDs. These substantially reduced optical gain thresholds are attributed to the large size of the QDs along with the gradient shell of the QDs, which helps to suppress the nonradiative $\mathrm{AR}^{24,25}$

Blue-emitting $\mathrm{CdZnS} / \mathrm{ZnS}$ QDs are synthesized using the slighlty modified recipe from the literature. ${ }^{26}$ Formation of the

Received: April 11, 2014

Accepted: June 6, 2014

Published: June 6, 2014 

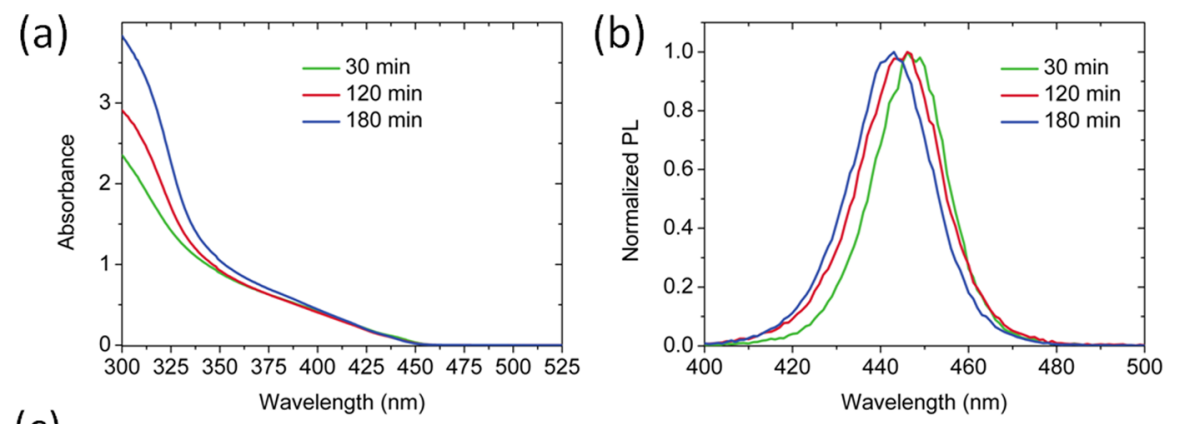

(c)
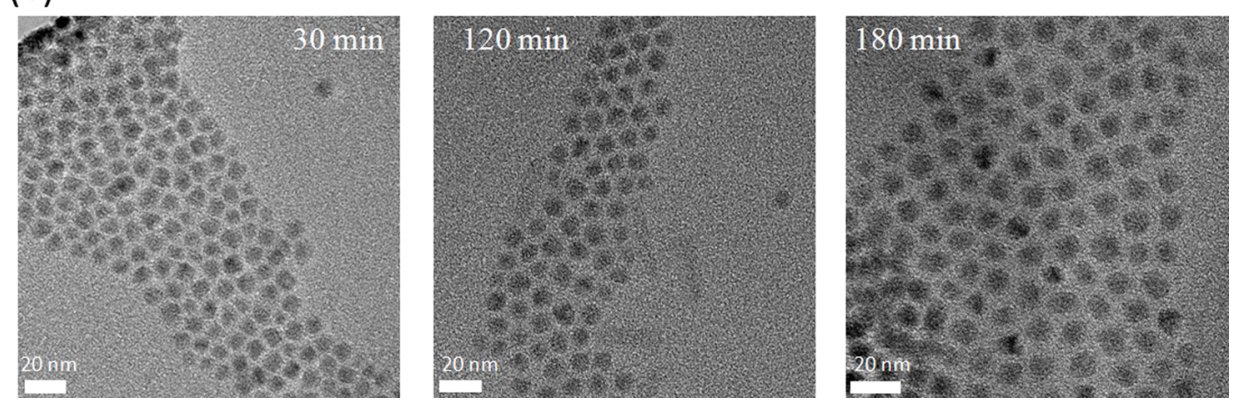

Figure 1. (a) Absorbance and (b) PL of the blue-emitting CdZnS/ZnS QDs having different shell growth times. (c) TEM images of the different duration shell-grown QDs: 30, 120, and $180 \mathrm{~min}$.

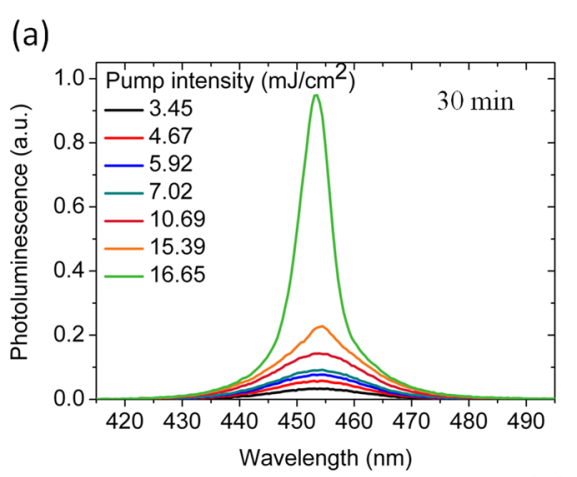

(b)

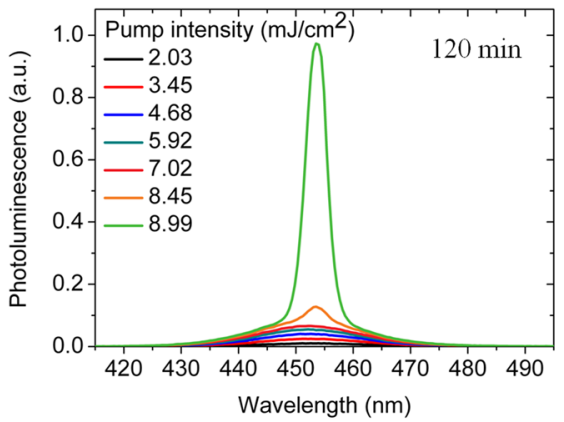

(d)

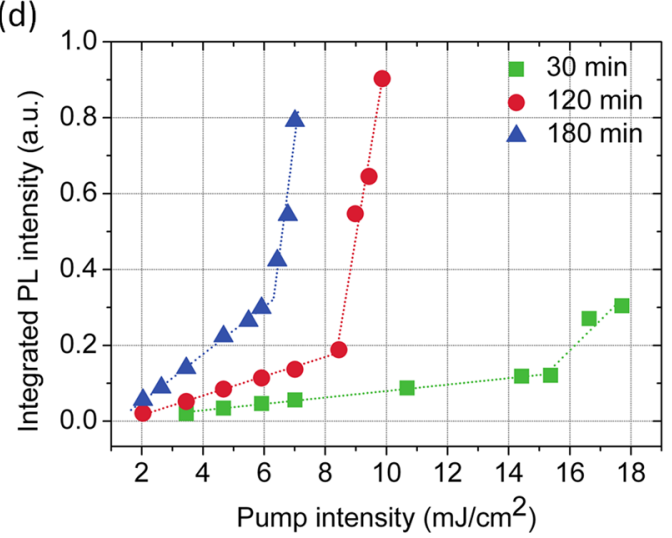

(c)

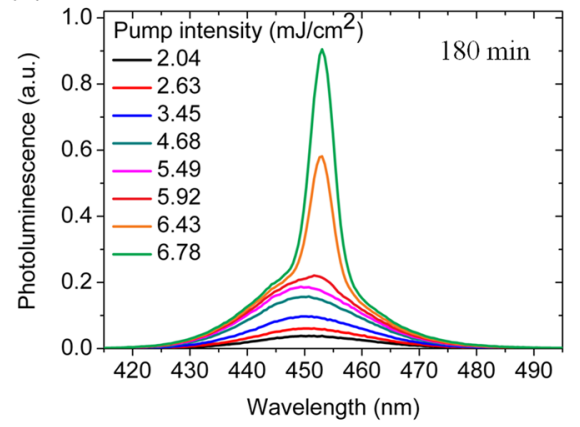

Figure 2. Pump-intensity-dependent PL of (a) 30, (b) 120, and (c) 180 min shell-grown CdZnS/ZnS QDs under 2PA pumping. (d) Luminescence versus pump intensity curve of the 30,120, and 180 min shell-grown QDs with 2PA pumping.

core and growth of the shell is carried out in a single-step synthesis. Sulfur injection is performed two times. With the first injection of the sulfur precursor, nucleation of $\mathrm{CdZnS}$ cores is achieved. Then, with the dropwise injection of the second sulfur precursor at later times, the shell growth is initiated. In this work, we study three different QD samples, which have the same $\mathrm{CdZnS}$ core but a varying duration for the shell growth (i.e., 30, 120, and $180 \mathrm{~min}$ ), resulting in different shell thickness and overall size (i.e., increasing size as the shell growth duration is increased). The absorption and the photoluminescence (PL) spectra of the QDs are shown in Figure 1a and b. The emission of the QDs in solution is located at $\sim 443-448 \mathrm{~nm}$. There is a slight blue shift of the spontenous emission peak as the shell growth duration is increased from 30 to $180 \mathrm{~min}$, which is an indication of the alloying and interdiffusion of $\mathrm{Zn}$ into the core/ shell interface. The size of the QDs is investigated by 
(a)

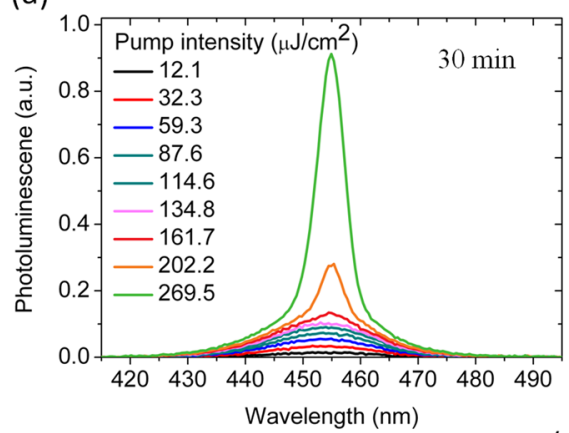

(b)

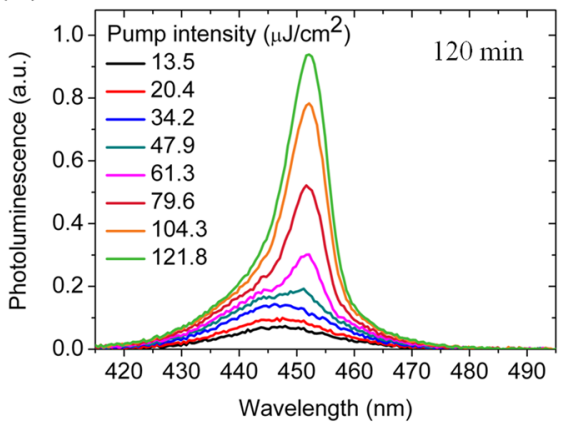

(c)

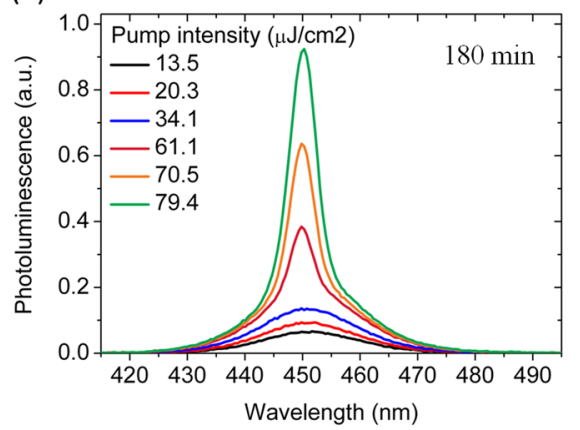

(d)

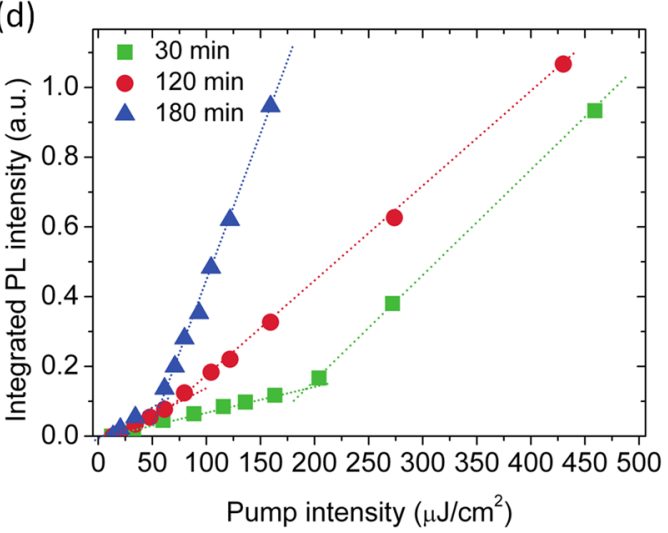

Figure 3. Pump-intensity-dependent PL of (a) 30, (b) 120, and (c) 180 min shell-grown CdZnS/ZnS QDs under 1PA pumping. (d) Luminescence versus pump intensity curve of the 180 min shell-grown QDs with 1PA pumping.

transmission electron microscopy (TEM). The analysis of the ensemble measurements reveals sizes of $8.36,9.15$, and 10.01 $\mathrm{nm}$ with a variance of $1 \mathrm{~nm}$ for the 30,120, and 180 min shellgrown QDs, respectively.

We study the optical gain performance of the solid films of the QDs that are spin-coated on quartz substrates at a high concentration $(50-60 \mathrm{mg} / \mathrm{mL}$ in hexane). As the pump source, we utilize a Spectra Physics-Spitfire Pro XP regenerative amplifier having a $120 \mathrm{fs}$ pulse width at $800 \mathrm{~nm}$ with $1 \mathrm{kHz}$ repetation rate. Using a cylindirical lens $(20 \mathrm{~cm}$ focus $)$, we excite the samples with a stripe geometry. Pump-intensitydependent PL is measured via collection of the emitted photons along the stripe using a fiber coupled to a spectrometer (Maya2000 Pro). For the case of 2PA-pumped films, pumpintensity-dependent PLs are shown for 30, 120, and $180 \mathrm{~min}$ shell-grown CdZnS/ZnS QDs in Figure $2 \mathrm{a}-\mathrm{c}$, respectively. At low pump intensities, spontenous emission dominates the PL spectra of the QDs that have full width at half-maxima (fwhm) of $20 \mathrm{~nm}$ for the $30 \mathrm{~min}$ QDs and $22 \mathrm{~nm}$ for the 120 and 180 min QDs. As the pump intensity is further increased, the PL spectrum starts to narrow down with an emerging ASE peak having a fwhm of $\sim 4.5-6.5 \mathrm{~nm}$. For the $30 \mathrm{~min}$ shell-grown QDs, the position of the ASE peak is almost at the same position as the spontaneous emission peak. For the 120 and 180 min shell-grown QDs, ASE is red-shifted by 2 and $2.3 \mathrm{~nm}$ as compared to the spontaneous emission PL peak of the QDs, respectively. Figure $2 \mathrm{~d}$ demonstrates the integrated PL intensity versus pump intensity for the three different QDs having different shell thicknesses. The thresholds for 2PA-pumped ASE are $6.25,8.44$, and $15.45 \mathrm{~mJ} / \mathrm{cm}^{2}$ for the 180,120 , and 30 min shell-grown QDs, respectively. Thus, we achieve a frequency upconverted stimulated emission in the QDs that has emission in the blue region of the visible spectrum. The
ASE threshold is comparable to the best-reported threshold for the red-emitting QDs and even better than that of the greenemitting QDs. ${ }^{12,13,17}$ The decrease of the ASE threshold as the shell thickness is increased is attributed to the increasing size of the QDs that both increase the 2PA cross section and also reduce the nonradiative $\mathrm{AR}$ together with the enhanced alloying of the core/shell interface. ${ }^{24,26,27}$ It was shown that QDs having a soft interface at the core-shell interface, that is, the gradient shell, have great contribution to the suppression of AR when compared to that for QDs having abrupt interfaces at the core-shell interace. ${ }^{24}$ With the combination of larger size and a gradient shell, suppressed AR and superior optical gain performances have been demonstrated from $\mathrm{CdSe} / \mathrm{CdS}$ core/ shell QDs. ${ }^{17,28}$ However, these types of QDs could be made to emit only in the red spectral range. Previously, it was not possible to achieve blue-emitting QDs from the gradient shell QDs (see Supporting Information for the depth profile study of the QDs using X-ray photoelectron spectroscopy revealing the gradient structure).

1PA-pumped ASE of these blue-emitting QDs is also investigated by frequency doubling the pump source using a nonlinear BBO crystal and filtering out the residual $800 \mathrm{~nm}$. Here, 180 min shell-grown QDs again exhibit the best ASE performance. Figure $3 a-c$ depicts the pump-intensity-dependent PL curves for the 30, 120, and $180 \mathrm{~min}$ shell-grown QDs, respectively. Interestingly, for the $180 \mathrm{~min}$ shell-grown QDs, the ASE peak emerges almost at the spontenous emission peak of the QDs $(\sim 450 \mathrm{~nm})$, whereas the ASE of the same QDs was achieved $\sim 2.3 \mathrm{~nm}$ red-shifted for the case of 2PA pumping. This could be attributed to the fact that $2 \mathrm{PA}$ pumping may cause extra heating that could red shift the emission. Another possibility is that the gain type might be altered from biexiton gain to single-exciton gain. ${ }^{8,9}$ Also, the ASE peak can move 
(a)

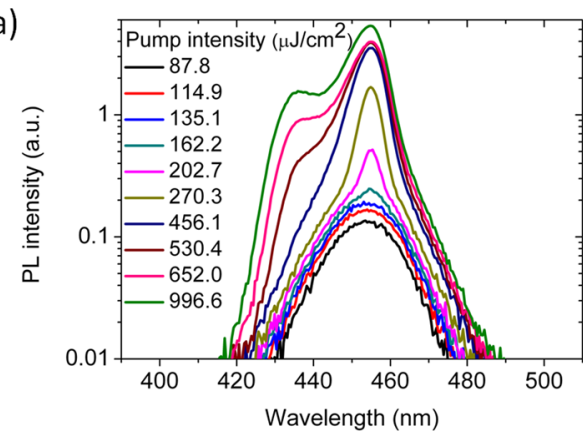

(b)

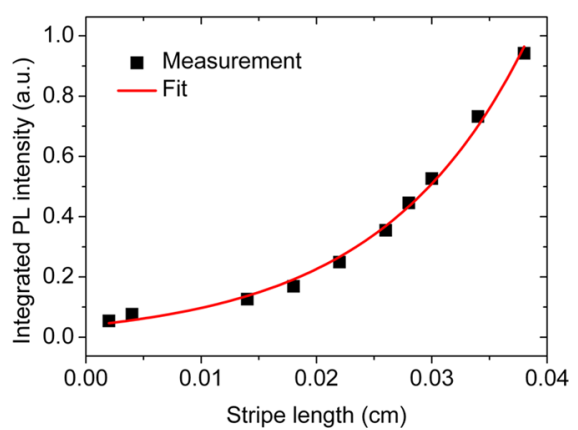

Figure 4. (a) PL intensity of the 30 min shell-grown QDs under 1PA pumping as a function of pump intensity. At higher pump intensities, higherorder multiexcitonic ASE is observed in addiation to the regular ASE. (b) Variable stripe length measurement of the gain coefficient of the 180 min shell grown QDs pumped by 1PA.

spectrally based upon surface trapping and/or multiexciton effects. ${ }^{17,21}$ For the 30 and 120 min QDs, we achieved the ASE peak red-shifted as compared to their spontenous emission peaks with a fwhm of 6-9 nm. Figure 3d shows the integrated emission intensity versus pump intensity curves for the three different QDs. The thresholds of the ASE for 1PA pumping are $58.9,74$, and $193.5 \mu \mathrm{J} / \mathrm{cm}^{2}$ for the 180,120 , and $30 \mathrm{~min}$ shell grown QDs, respectively. The 1PA ASE threshold achieved for the $180 \mathrm{~min}$ QDs represents an order of magnitude better result than the previously reported best threshold for the blueemitting QDs, which was $\sim 800 \mu \mathrm{J} / \mathrm{cm}^{2}$. . The 1PA ASE threshold of our blue QDs is comparable to that of the state-ofthe art green- and red-emitting QD-based gain media. ${ }^{8}$ Recently, using similar QDs, record efficiency electroluminescent light-emitting diodes were shown, where the enhanced performance was attributed to the suppression of the nonradiative $\mathrm{AR}$ processes and inter- $\mathrm{QD}$ nonradiative energy transfer. ${ }^{26}$

Furthermore, we investigate the emission of our QDs at increased pump intensities to understand the suppression of the nonradiative AR. Surprisingly, we observe higher-order multiexcitonic ASE for the first time using blue-emitting QDs. ${ }^{29}$ This type of higher-order ASE could be only shown previously for red-emitting core/shell QDs, ${ }^{30}$ dot-in-rod, and tetrapod-like nanocrystals. $^{31}$ Figure $4 \mathrm{a}$ shows the pump intensity (1PApumped) dependent PL of the 30 min shell-grown QDs. As the pump intensity is below the ASE threshold, we observe the spontenous emission of the QDs. After surprassing the ASE threshold $\left(\sim 200 \mu \mathrm{J} / \mathrm{cm}^{2}\right)$, the ASE peak emerges and starts to dominate the PL spectra. At further increased pump intensity $\left(530 \mu \mathrm{J} / \mathrm{cm}^{2}\right)$, which is 2.5 -fold greater than the ASE threshold, we observe the onset of a new emission peak at the higherenergy side of the spontaneous emission peak $(110 \mathrm{meV}$ blueshifted as compared to the spontaneous emission peak). At further increased pump intensities, this newly emerging peak increases at a faster rate than the regular ASE peak. We attribute this newly emerging higher-energy peak to the ASE resulting from higher-order excitonic states $\left(1 \mathrm{P}_{\mathrm{e}}-1 \mathrm{P}_{3 / 2}\right)$ in these QDs. This observation of higher-order excitonic ASE supports the existence of the long-lived multiexitons, which is a clear indication of the suppressed nonradiative AR. Otherwise, higher-order excitons would not be sustained in these QDs. This type of multiexcitonic ASE could not be observed for the blue-emitting QDs before. Finally, we measure the gain coefficient of our blue-emitting QDs, as shown in Figure $4 \mathrm{~b}$ by applying the variable stripe length method. The integrated PL intensity of the $180 \mathrm{~min}$ shell-grown QDs is shown in
Figure $4 \mathrm{~b}$ for varying stripe length (VSL). ${ }^{32}$ The VSL data is fitted according to ref 33 . The gain coefficient turns out to be up to $78 \mathrm{~cm}^{-1}$. This value is comparable to the highest reported gain coefficient of $150 \mathrm{~cm}^{-1}$ for the red-emitting QDs. ${ }^{34}$

As a summary, we synthesize and study optical gain performance of $\mathrm{CdZnS} / \mathrm{ZnS}$ QDs having both large size and a gradient shell that emits in the blue spectral region. For the first time, 2PA-pumped ASE is shown with a threshold as low as $\sim 6 \mathrm{~mJ} / \mathrm{cm}^{2}$ for the blue-emitting QDs. Furthermore, these QDs exhibited the best-reported 1PA-pumped ASE performance with a threshold down to $60 \mu \mathrm{J} / \mathrm{cm}^{2}$ and gain coefficient up to $\sim 80 \mathrm{~cm}^{-1}$. These QDs will be expected to be promising optical gain media for blue-emitting QD-based lasers.

\section{ASSOCIATED CONTENT}

\section{Supporting Information}

Details of a depth profile study of the quantum dots using X-ray photoelectron spectroscopy. This material is available free of charge via the Internet at http://pubs.acs.org.

\section{AUTHOR INFORMATION}

\section{Corresponding Author}

*E-mail: volkan@bilkent.edu.tr or hvdemir@ntu.edu.sg.

\section{Author Contributions}

${ }^{\#}$ B.G., Y.K., and M.Z.A. contributed equally to this work.

\section{Notes}

The authors declare no competing financial interest.

\section{ACKNOWLEDGMENTS}

The authors would like to thank the financial support from EUFP7 Nanophotonics4Energy NoE and TUBITAK EEEAG 109E002, 109E004, 110E010, 110E217, NRF-RF-2009-09, NRF-CRP-6-2010-02, and A*STAR of Singapore. H.V.D. acknowledges support from ESF-EURYI and TUBA-GEBIP.

\section{REFERENCES}

(1) Murray, C. B.; Norris, D. J.; Bawendi, M. G. Synthesis and Characterization of Nearly Monodisperse CdE (E = Sulfur, Selenium, Tellurium) Semiconductor Nanocrystallites. J. Am. Chem. Soc. 1993, 115, 8706-8715.

(2) Peng, X.; Manna, L.; Yang, W.; Wickham, J.; Scher, E.; Kadavanich, A.; Alivisatos, A. Shape Control of CdSe Nanocrystals. Nature 2000, 404, 59-61.

(3) Coe, S.; Woo, W.-K.; Bawendi, M.; Bulović, V. Electroluminescence from Single Monolayers of Nanocrystals in Molecular Organic Devices. Nature 2002, 420, 800-803. 
(4) Klimov, V. I.; Mikhailovsky, A. A.; Xu, S.; Malko, A.; Hollingsworth, J. A.; Leatherdale, C. A.; Eisler, H.; Bawendi, M. G. Quantization of Multiparticle Auger Rates in Semiconductor Quantum Dots. Science 2000, 290, 314-317.

(5) Eisler, H. H.-J.; Sundar, V. V. C.; Bawendi, M. G.; Walsh, M.; Smith, H. I.; Klimov, V. Color-Selective Semiconductor Nanocrystal Laser. Appl. Phys. Lett. 2002, 80, 4614.

(6) Chan, Y.; Steckel, J. S.; Snee, P. T.; Caruge, J.-M.; Hodgkiss, J. M.; Nocera, D. G.; Bawendi, M. G. Blue Semiconductor Nanocrystal Laser. Appl. Phys. Lett. 2005, 86, 073102.

(7) Chen, Y.; Guilhabert, B.; Herrnsdorf, J.; Zhang, Y.; Mackintosh, A. R.; Pethrick, R. A.; Gu, E.; Laurand, N.; Dawson, M. D. Flexible Distributed-Feedback Colloidal Quantum Dot Laser. Appl. Phys. Lett. 2011, 99, 241103.

(8) Dang, C.; Lee, J.; Breen, C.; Steckel, J. S.; Coe-Sullivan, S.; Nurmikko, A. Red, Green and Blue Lasing Enabled by Single-Exciton Gain in Colloidal Quantum Dot Films. Nat. Nanotechnol. 2012, 7, 335-339.

(9) Klimov, V. I.; Ivanov, S. A.; Nanda, J.; Achermann, M.; Bezel, I.; McGuire, J. A.; Piryatinski, A. Single-Exciton Optical Gain in Semiconductor Nanocrystals. Nature 2007, 447, 441-446.

(10) Kambhampati, P. Multiexcitons in Semiconductor Nanocrystals: A Platform for Optoelectronics at High Carrier Concentration. J. Phys. Chem. Lett. 2012, 3, 1182-1190.

(11) Jasieniak, J.; Pacifico, J.; Signorini, R.; Chiasera, A.; Ferrari, M.; Martucci, A.; Mulvaney, P. Luminescence and Amplified Stimulated Emission in CdSe-ZnS-Nanocrystal-Doped $\mathrm{TiO}_{2}$ and $\mathrm{ZrO}_{2}$ Waveguides. Adv. Funct. Mater. 2007, 17, 1654-1662.

(12) Jasieniak, J. J. J.; Fortunati, I.; Gardin, S.; Signorini, R.; Bozio, R.; Martucci, A.; Mulvaney, P. Highly Efficient Amplified Stimulated Emission from CdSe-CdS-ZnS Quantum Dot Doped Waveguides with Two-Photon Infrared Optical Pumping. Adv. Mater. 2008, 20, 69-73.

(13) Signorini, R.; Fortunati, I.; Todescato, F.; Gardin, S.; Bozio, R.; Jasieniak, J. J.; Martucci, A.; Della Giustina, G.; Brusatin, G.; Guglielmi, M.; et al. Facile Production of Up-Converted Quantum Dot Lasers. Nanoscale 2011, 3, 4109-4113.

(14) Fortunati, I.; Signorini, R.; Bozio, R.; Jasieniak, J. J.; Antonello, A.; Martucci, A.; Della Giustina, G.; Brusatin, G.; Guglielmi, M. CdSe Core-Shell Nanoparticles as Active Materials for Up-Converted Emission. J. Phys. Chem. C 2011, 115, 3840-3846.

(15) Zhang, C.; Zhang, F.; Cheng, A.; Kimball, B.; Wang, A. Y. Frequency Upconverted Lasing of Nanocrystal Quantum Dots in Microbeads Frequency Upconverted Lasing of Nanocrystal Quantum Dots in Microbeads. Appl. Phys. Lett. 2009, 95, 183109/1-183109/3.

(16) Xing, G.; Liao, Y.; Wu, X.; Chakrabortty, S.; Liu, X.; Yeow, E. K. L.; Chan, Y.; Sum, T. C. Ultralow-Threshold Two-Photon Pumped Amplified Spontaneous Emission and Lasing from Seeded CdSe/CdS Nanorod Heterostructures. ACS Nano 2012, 6, 10835-10844.

(17) Cihan, A. F.; Kelestemur, Y.; Guzelturk, B.; Yerli, O.; Kurum, U.; Yaglioglu, H. G.; Elmali, A.; Demir, H. V. Attractive versus Repulsive Excitonic Interactions of Colloidal Quantum Dots Control Blue- to Red-Shifting (and Non-Shifting) Amplified Spontaneous Emission. J. Phys. Chem. Lett. 2013, 4, 4146-4152.

(18) Ivanov, S. A.; Nanda, J.; Piryatinski, A.; Achermann, M.; Balet, L. P.; Bezel, I. V.; Anikeeva, P. O.; Tretiak, S.; Klimov, V. I. Light Amplification Using Inverted Core/Shell Nanocrystals: Towards Lasing in the Single-Exciton Regime. J. Phys. Chem. B 2004, 108, 10625-10630.

(19) Darugar, Q.; Qian, W.; El-Sayed, M. A. Observation of Optical Gain in Solutions of CdS Quantum Dots at Room Temperature in the Blue Region. Appl. Phys. Lett. 2006, 88, 261108.

(20) Cooney, R.; Sewall, S.; Sagar, D.; Kambhampati, P. Gain Control in Semiconductor Quantum Dots via State-Resolved Optical Pumping. Phys. Rev. Lett. 2009, 102, 127404.

(21) Cooney, R. R.; Sewall, S. L.; Sagar, D. M.; Kambhampati, P. State-Resolved Manipulations of Optical Gain in Semiconductor Quantum Dots: Size Universality, Gain Tailoring, and Surface Effects. J. Chem. Phys. 2009, 131, 164706.
(22) Sewall, S.; Franceschetti, A.; Cooney, R.; Zunger, A.; Kambhampati, P. Direct Observation of the Structure of Band-Edge Biexcitons in Colloidal Semiconductor CdSe Quantum Dots. Phys. Rev. B 2009, 80, 081310.

(23) Sewall, S. L.; Cooney, R. R.; Dias, E. A.; Tyagi, P.; Kambhampati, P. State-Resolved Observation in Real Time of the Structural Dynamics of Multiexcitons in Semiconductor Nanocrystals. Phys. Rev. B 2011, 84, 235304.

(24) Cragg, G. E.; Efros, A. L. Suppression of Auger Processes in Confined Structures. Nano Lett. 2010, 10, 313-317.

(25) Wang, X.; Ren, X.; Kahen, K.; Hahn, M. A.; Rajeswaran, M.; Maccagnano-Zacher, S.; Silcox, J.; Cragg, G. E.; Efros, A. L.; Krauss, T. D. Non-Blinking Semiconductor Nanocrystals. Nature 2009, 459, 686-689.

(26) Lee, K.-H.; Lee, J.-H.; Song, W.; Ko, H.; Lee, C.; Lee, J.; Yang, H. Highly Efficient, Color-Pure, Color-Stable Blue Quantum Dot Light-Emitting Devices. ACS Nano 2013, 7, 7295-7302.

(27) Bae, W. K.; Padilha, L. A.; Park, Y.-S.; McDaniel, H.; Robel, I.; Pietryga, J. M.; Klimov, V. I. Controlled Alloying of the Core-Shell Interface in $\mathrm{CdSe} / \mathrm{CdS}$ Quantum Dots for Suppression of Auger Recombination. ACS Nano 2013, 7, 3411-3419.

(28) García-Santamaría, F.; Chen, Y.; Vela, J.; Schaller, R. D.; Hollingsworth, J. A.; Klimov, V. I. Suppressed Auger Recombination in "Giant" Nanocrystals Boosts Optical Gain Performance. Nano Lett. 2009, 9, 3482-3488.

(29) Kambhampati, P. Unraveling the Structure and Dynamics of Excitons in Semiconductor Quantum Dots. Acc. Chem. Res. 2011, 44, $1-13$.

(30) Chan, Y.; Caruge, J.-M.; Snee, P. T.; Bawendi, M. G. Multiexcitonic Two-State Lasing in a CdSe Nanocrystal Laser. Appl. Phys. Lett. 2004, 85, 2460.

(31) Liao, Y.; Xing, G.; Mishra, N.; Sum, T. C.; Chan, Y. Low Threshold, Amplifi Ed Spontaneous Emission from Core-Seeded Semiconductor Nanotetrapods Incorporated into a Sol-Gel Matrix. Adv. Mater. 2012, 24, 159-164.

(32) Shaklee, K. L.; Nahory, R. E.; Leheny, R. F. Optical Gain in Semiconductors. J. Lumin. 1973, 7, 284-309.

(33) Samuel, I. D. W.; Turnbull, G. A. Organic Semiconductor Lasers. Chem. Rev. 2007, 107, 1272-1295.

(34) Malko, A. V.; Mikhailovsky, A. A.; Petruska, M. A.; Hollingsworth, J. A.; Htoon, H.; Bawendi, M. G.; Klimov, V. I. From Amplified Spontaneous Emission to Microring Lasing Using Nanocrystal Quantum Dot Solids. Appl. Phys. Lett. 2002, 81, 1303. 